\title{
Modularity Index Metrics for Java-Based Open Source Software Projects
}

\author{
Andi Wahju Rahardjo Emanuel \\ Informatics Bachelor Program, \\ Faculty of Information Technology, \\ Maranatha Christian University, \\ Bandung, Indonesia
}

\author{
Retantyo Wardoyo, Jazi Eko Istiyanto, \\ Khabib Mustofa \\ Dept. of Computer Science and Electronics, \\ Universitas Gadjah Mada, \\ Yogyakarta, Indonesia
}

\begin{abstract}
Open Source Software (OSS) Projects are gaining popularity these days, and they become alternatives in building software system. Despite many failures in these projects, there are some success stories with one of the identified success factors is modularity. This paper presents the first quantitative software metrics to measure modularity level of Java-based OSS Projects called Modularity Index. This software metrics is formulated by analyzing modularity traits such as size, complexity, cohesion, and coupling of 59 Java-based OSS Projects from sourceforge.net using SONAR tool. These OSS Projects are selected since they have been downloaded more than $100 \mathrm{~K}$ times and believed to have the required modularity trait to be successful. The software metrics related to modularity in class, package and system level of these projects are extracted and analyzed. The similarities found are then analyzed to determine the class quality, package quality, and then combined with system architecture measure to formulate the Modularity Index. The case study of measuring Modularity Index during the evolution of JFreeChart project has shown that this software metrics is able to identify strengths and potential problems of the project.
\end{abstract}

Keywords-Open source software projects; modularity; Java; sourceforge; software metrics; system architecture.

\section{INTRODUCTION}

Open Source Software (OSS) Projects are gaining popularity these days. They were once only considered as an experimental way of academics and researchers to share the programming experiences, now they become the mainstream software development methodology comparable to those of commercial and proprietary software projects. This movement was initially started by Richard Stallman [33] and Eric Raymond [31]. Some success stories of OSS Projects include Linux Operating System, Apache Web Server, Mozilla Web Browser, LibreOffice, etc. The success of these projects is attributed to many key success factors such as the fact that the developer is the actual user [10], and sound and modular architecture [20][17][11], the existence of communities that support the system development [9], etc. From all these success factors, modularity of the software system is one of the important factors to be examined further in this paper.

Even though there are some proofs of the success of OSS Projects, some facts that many more similar projects are unsuccessful or failed also unavoidable exist [16]. There are some characteristics of OSS Projects that have been identified contributing to such unfruitful result such as no formal means i.e. no project planning [4], poor coding styles of project initiators [13] and poor architectural design [12]. We believe that some new approaches with respect to modularity to counter such problems in OSS Projects are needed. Until now, modularity has been identified as a key success factor of OSS projects, but how to apply modularity, especially from early phase of the project is not yet understood.

This paper presents the formulation of Modularity Index which is the first quantitative software metrics to measure the modularity level in OSS Projects.

This paper is organized as follows: section 2 describes the recent studies in OSS Projects, modularity in OSS Projects and Software Metrics. Section 3 describes the data source of OSS Projects for analysis. Section 4 shows the step by step Modularity Index formulation starts from class level, package level, and system level. The case study of 33 out of 52 versions of JFreeChart projects is shown in section 5. Finally, section 6 describes the conclusion of the paper and future studies of the research.

\section{RECENT STUDIES}

\section{A. OSS Projects}

Many web portals have been developed as an incubator for OSS Project's developers to develop and host their projects. These portals are equipped with many development tools and statistics to assist the project initiator or administrator in improving their projects and other interested contributors to join the projects. Some of the popular portals are Sourceforge.net, freshmeat.net, launchpad.net, and Google Code.

The OSS Projects themselves have several distinct characteristics not found in commercial / proprietary software development [10][26], which are:

- The source code of the application is freely available for everybody to download, improve and modify [31].

- People who contribute to the development of the OSS projects are usually forming a group called communities. The recruitment process if this groups are completely voluntary [9]. This communities is an example of true merit-based system of hierarchy [11] 
- The development methods of the projects are lacking of formal methodology found in commercially developed software applications [4]. The two most important activities are fixing bugs and adding features [3].

There are already many studies relating to OSS Projects that are classified into three main categories. The first category is the study of large and successful OSS Projects to find their success characteristics such as Debian [32], FreeBSD [12], Apache [27], Open BSD [22], and many more. The second category is the study to find similarities in several OSS Projects such as Apache dan Mozilla [26], 15 OSS Projects [35], and 2 OSS Projects [6]. The last category is the study on the process aspects in OSS Projects such as Requirement Engineering [30], code fault [22], Design Pattern [18], reliability model [37], phase of development [34], and work practice in OSS projects [10].

Current studies about OSS Projects mostly focus on the already successful and large projects that have already established hierarchy and system, while most of the failed and unsuccessful OSS Projects are usually small or medium sized projects [16]. The application of these hierarchy and system in already established projects into small to medium sized projects may not be suitable. In our initial research, we have conducted analysis on more than $130 \mathrm{~K}$ OSS Projects to find their success factors [15].

\section{B. Modularity in OSS Projects}

Modularization involves breaking up of an software system into smaller, more independent elements known as module [23]. Booch has defined modularity as the property of a system whose modules are cohesive and loosely-coupled [24]. Fenton stated that modularity is the internal quality attribute of the software system [24]. It is also known that modularity is directly related to software architecture, since modularity is separation of a software system in independent and collaborative modules that can be organized in software architecture [29]. Modular software has several advantages such as maintainability, manageability, and comprehensibility [28]. Moreover, modularity has been identified as one of the key success factors in OSS Projects [20][17][11].

There are five attributes closely related to modularity in software system which are coupling / dependency, complexity, cohesion, and information hiding [21][7]. To have an ideal modular software system, the system should have the following attributes:

- Small size in each module (package) and many modules in the system [36]: each module / package should only responsible for simple feature, and the more complex features should be composed of many of these simple features. The possible software metrics to measure size are NCLOC (non-commenting lines of code), Lines, or Statements.

- Low coupling / dependency [5]: minimization or standardization of coupling / dependency e.g. through standard format i.e. published APIs [2], elimination of semantic dependencies, etc.
- Low complexity: hierarchy of modules that prefers flatter than taller dependency [28][2].

- High cohesion [21]: high integrity of the internal structure of software modules which is usually stated as either high cohesion or low cohesion.

- Open for extension and close to modification [5]: capability of the existing module to be extended to create a more complex module. And avoid changing already debugged code. The creation of new modules should be encourage using available extension and not modifying the already tested module.

Even though modularity is already identified as the key success factor in OSS Projects, the justification for it in large and succesful OSS Projects is purely qualitative. The software metrics attributing to the modularity properties are all separated and not yet integrated into a single measure. This paper will present a single measure called Modularity Index that quantitatively determines the modularity level of OSS Projects.

\section{Software Metrics}

Software metrics are defined as certain values which are expressed in some units attributed to software application [25]. The software metrics are useful in indicate the current state of the software and enable to compare and predict the current achievement of software applications [25]. There are several known software metrics based on its categories [25]:

- Size-related software metrics: NCLOC, Memory footprint, Number of classes / headers, Number of methods, Number of attributes, Size of compiled code, etc.

- Quality-related software metrics: Cyclomatic complexity, Number of states, Number of bugs in LOC, Coupling metrics, Inheritance metrics, etc.

- Process-related software metrics: failed builds, defect per hour, requirement changes, programming time, number of patches after release, etc.

There are currently more than 200 metrics with many different purposes [25], and one of the study by the authors are the statistical analysis of software metrics affecting modularity in OSS Projects [14].

\section{DATA SOURCE OF OSS PROJECTS}

The data source of the OSS Projects for the experiment is from the sourceforge.net portal since it is the largest OSS Portal.

\section{A. Assumptions and Considerations}

There are several consideration and assumption in selecting which OSS Projects to be analyzed, which are:

- The OSS projects are build using Java programming language, and a single package in the project resembles a "module" in modular software system. The addition of package in the software is intended as the addition of new feature in the system. 
- The project's size is limited to small-to-medium-sized OSS Projects. The limitation of the size (NCLOC) of OSS Projects being evaluated are $170 \mathrm{~K}$. The concept of modularity is a lot easier to comprehend in objectoriented programming language (i.e. $\mathrm{C}++$, Java, etc.) compared to procedural programming (i.e. C, Fortran, etc.), since the concept of module, coupling, cohesion, etc. are more straightforward. Java-based OSS Projects are selected since they are among the mostly popular object oriented programming for developing Open Source Software [16].

- The Projects should already be downloaded more than 100,000 times. This high number of downloads may indicate the "success" of the projects, which in turn may imply modularity traits that already identified as the success factor of OSS Project [20][17][11].

- The source code of the OSS Project is syntax error-free and compile-able. The SONAR tool requires that the source code should be compiled first using compile tool such as maven, or ant. Many of the OSS Projects provides separate binary and source code and it is difficult to create binary directly from the source code due to several reasons such as compile error, build tool configuration error, syntax error, etc.

\section{B. Selected OSS Projects}

Table 1. shows the list of OSS Projects as a subject for this research. The initial OSS Projects to be evaluated are 209 projects, but only 59 which are suitable to be evaluated using SONAR due to the assumptions and considerations stated in section III.A. There are total 1885 modules / packages being measured from these 59 OSS Projects.

TABLE I. LIST OF 59 SELECTED OSS PROJECTS

\begin{tabular}{|l|l|l|l|}
\hline No & \multicolumn{1}{|c|}{ Project Name } & No & \multicolumn{1}{|c|}{ Project Name } \\
\hline 1 & FreeMind & 31 & Jin client for chess servers \\
\hline 2 & jEdit & 32 & SAX: Simple API for XML \\
\hline 3 & TV-Browser - A free EPG & 33 & jKiwi \\
\hline 4 & JFreeChart & 34 & Data Crow \\
\hline 5 & JasperReports - Java Reporting & 35 & Wicket \\
\hline 6 & $\begin{array}{l}\text { OpenProj } \\
\text { Management }\end{array}$ & 36 & Cewolf - Chart TagLib Project \\
\hline 7 & HyperSQL Database Engine & 37 & DrawSWF \\
\hline 8 & yura.net & 38 & $\begin{array}{l}\text { c3p0:JDBC DataSources } \\
\text { / Resource Pools }\end{array}$ \\
\hline 9 & JabRef & 39 & JavaGroups \\
\hline 10 & FreeCol & 40 & $\begin{array}{l}\text { OmegaT - multiplatform CAT } \\
\text { tool }\end{array}$ \\
\hline 11 & jTDS - SQL Server and Sybase & 41 & FreeGuide TV Guide \\
\hline 12 & TDBC driver & 42 & Eteria IRC Client \\
\hline 13 & FindBugs & 43 & MeD's Movie Manager \\
\hline 14 & PMD & 44 & subsonic \\
\hline 15 & JGraph Diagram Component & 45 & kXML \\
\hline
\end{tabular}

\begin{tabular}{|l|l|c|l|}
\hline No & \multicolumn{1}{|c|}{ Project Name } & No & \multicolumn{1}{|c|}{ Project Name } \\
\hline 16 & ANts P2P & 46 & Jaxe \\
\hline 17 & Paros & 47 & The JUMP Pilot Project \\
\hline 18 & $\begin{array}{l}\text { ProGuard Java Optimizer and } \\
\text { Obfuscator }\end{array}$ & 48 & $\begin{array}{l}\text { Aglet Software Development } \\
\text { Kit }\end{array}$ \\
\hline 19 & TripleA & 49 & Antenna \\
\hline 20 & JSch & 50 & CBViewer \\
\hline 21 & Jajuk & 51 & Sunflow Rendering System \\
\hline 22 & FreeTTS & 52 & Thingamablog \\
\hline 23 & $\begin{array}{l}\text { A Java } \\
\text { reading/writing Excel }\end{array}$ & 53 & BORG Calendar \\
\hline 24 & checkstyle & 54 & $\begin{array}{l}\text { Directory Synchronize Pro } \\
\text { (DirSync Pro) }\end{array}$ \\
\hline 25 & httpunit & 55 & Java Treeview \\
\hline 26 & JMSN & 56 & Java Network Browser \\
\hline 27 & PDFBox & 57 & Red Piranha \\
\hline 28 & JBidwatcher & 58 & Cobertura \\
\hline 29 & JTidy & 59 & Jake2 \\
\hline 30 & Jena & - & - \\
\hline & & \\
\hline
\end{tabular}

\section{Steps}

In order to be able to analyze these OSS Projects, there are some steps being performed, which are:

- Compile the source code using available build tool (Ant or Maven2).

- Execute maven2 script to start analyze the OSS Projects using SONAR tool.

- Creating custom portal to perform the required analysis.

- Analyze and find the correlation and similarities of all the projects such as using scatter graph, least square fit, histogram, etc.

\section{MODUlaRITy INDEX FORMULATION}

The formulation of modularity index will start from the class level, then move up to the package level, and finally concluded in the system level.

\section{A. Class Level Modularity}

There are four software metrics that determine the level of modularity in class level, which are:

- Size Metrics which consists of: NCLOC, Lines, and Statements. NCLOC is the number of non-commenting lines of code. The selection of NCLOC will also represent the other size metrics [14].

- Cohesion: LCOM4 or Lack of Cohesion Method version 4, this version is better for object oriented programming such as Java as proposed by Hitz and Montazeri [19] which is the improvement of LCOM1 Chidamber and Kemerer [8].

- Complexity: McCabe's Cyclomatic Complexity [22] is one example of complexity metrics that widely used. 
Our previous paper have shown that the size metrics and complexity metrics are highly related so this metrics may be ignored [14].

- Functions: the number of functions / methods in the class. This may indicates the complexity

1) NCLOC: Figure 1 shows the histogram of the class vs. NCLOC of the all OSS Projects being evaluated. The value of NCLOC peaked at 50 with the histogram before the peak resembles linear straight line and after the peak resembles inverse polynomial line. The value of approximation of both lines are shown in the Fig.1.

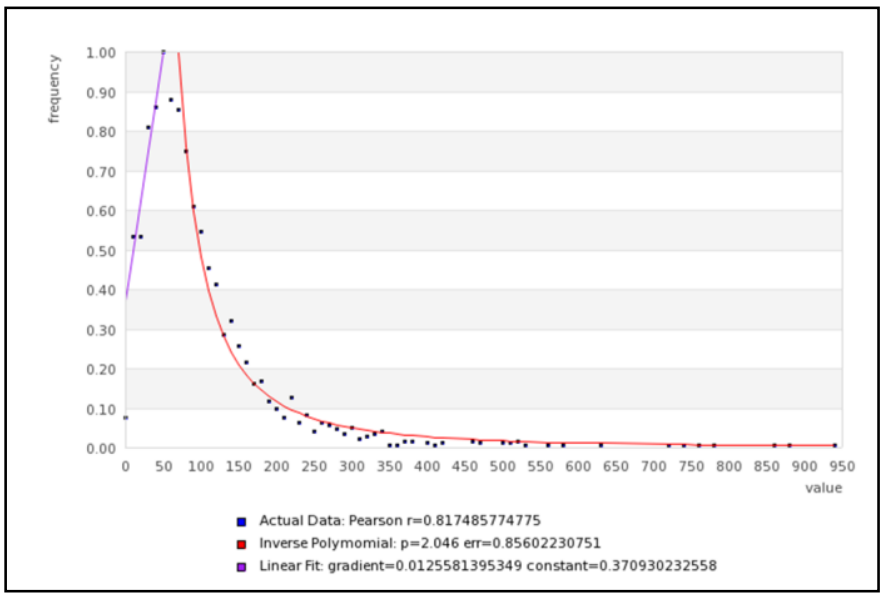

Figure 1. Histogram of Classes vs. NCLOC

If $L O C_{Q}$ is defined as the normalized value of the quality of $\mathrm{NCLOC}$, so the formula of $\mathrm{LOC}_{\mathrm{Q}}$ are:

$$
\begin{gathered}
\mathrm{LOC}_{\mathrm{Q}}=0.0125 \times \mathrm{NCLOC}+0.375 \text { for } \mathrm{NCLOC} \leq 50 \\
\mathrm{LOC}_{\mathrm{Q}}=(\mathrm{NCLOC}-50)^{-2.046} \text { for } \mathrm{NCLOC}>50
\end{gathered}
$$

Where:

$$
\begin{aligned}
& \text { LOC }_{Q}=\text { NCLOC Quality Value } \\
& \text { NCLOC }=\text { NCLOC Value }
\end{aligned}
$$

Note: the value of constant in formula (1) is adjusted from 0.371 into 0.375 to achieve the maximum value of 1 at NCLOC $=50$.

2) Number of Functions: Figure 2 shows the histogram of classes vs. functions of all OSS Projects being evaluated. The peak value is 4.83 (rounded up into 5). Similar to class vs. NCLOC, the values before the peak resembles a straight line and after the peak resembles an inverse polynomial line with the approximation of both lines shown in the Fig. 2.

$\mathrm{F}_{\mathrm{Q}}$ is defined as the normalized value of function's quality, it can be formulated as follows:

$$
\begin{gathered}
F_{Q}=0.172 \times F+0.171 \text { for } F \leq 5 \\
F_{Q}=(F-4.83)^{-2.739} \text { for } F>5
\end{gathered}
$$

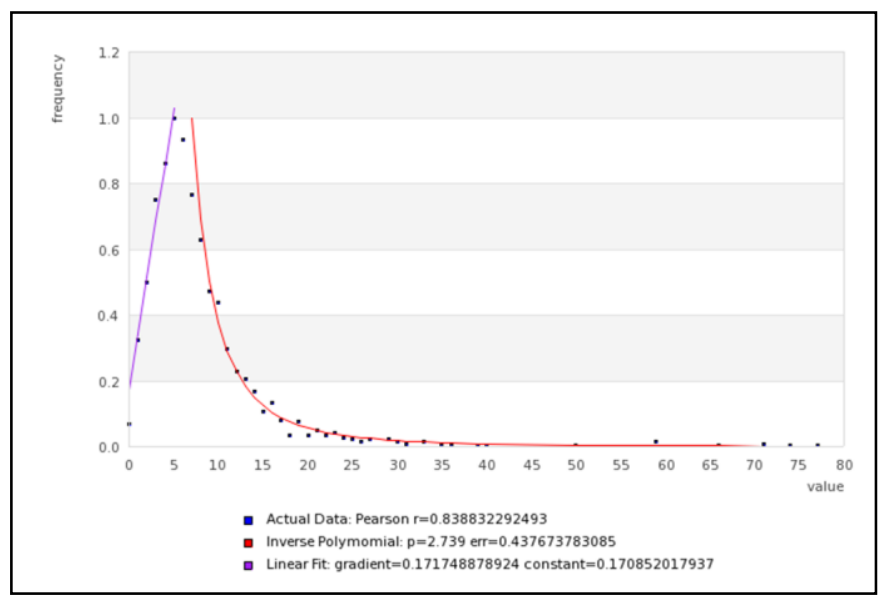

Figure 2. Histogram of Classes vs. Functions

Where:

$$
\begin{array}{ll}
\mathrm{F}_{\mathrm{Q}} & =\text { Function Quality Value } \\
\mathrm{F} & =\text { Number of Function }
\end{array}
$$

3) Cohesion: Cohesion is determined by the value of LCOM4. The ideal value is 1 which means that the class is highly cohesive. Higher value of LCOM4 indicates the degree of needed separation of classes into smaller classes.

$$
\text { LCOM4 } \geq 1
$$

Where:

$$
\text { LCOM4 = Class Cohesion Value }
$$

4) Class Quality Formulation: Integrating all above measures into a single normalized value, the formulation of class quality or $\mathrm{C}_{\mathrm{Q}}$ are:

$$
C_{Q}=\frac{L O C_{Q}+F_{Q}}{2 \times L C O M 4}
$$

Where:

$C_{Q} \quad=$ Class Quality Value

LOC $_{\mathrm{Q}}=$ NCLOC Quality Value

$\mathrm{F}_{\mathrm{Q}} \quad=$ Function Quality Value

LCOM4 = Class Cohesion Value

\section{B. Package Level Modularity}

Package Quality or $\mathrm{P}_{\mathrm{Q}}$ is the quality of individual package. Since in a single package there are many classes and there is no similarities found the the optimal number of classes in each package, so the Package Quality is determined by the average Class Quality or stated as:

$$
\mathrm{P}_{\mathrm{Q}}=\operatorname{avg}\left(C_{Q}\right)
$$

Where: 


$$
\begin{aligned}
& \mathrm{P}_{\mathrm{Q}}=\text { Package Quality Value } \\
& C_{Q}=\text { Class Quality Value }
\end{aligned}
$$

\section{System Level Modularity}

$\mathrm{S}_{\mathrm{A}}$ is a normalized value (with maximum value of 1 ) which determine the value of software architecture. The factors that influence this value are Package Cohesion (relationship among classes within package) and Package Coupling (relationship among classes from different packages). The principle used here is "Maximize Cohesion and Minimize Coupling" which becomes a widely known principle in building a good software system. The form of formulation is based on presentation titled "Software Architecture Metrics" by Ammar et. al [1], with the difference is that instead of using entropy approaches, this formulation is using the actual value of dependencies in determining the value of Package Cohesion and Package Coupling.

$$
S_{A}=\sqrt{\frac{\sum_{i=1}^{d} C_{i i}{ }^{2}}{\sum_{i=1}^{d} \sum_{j=1}^{d} C_{i j}{ }^{2}}}
$$

Where:

$$
\begin{aligned}
\mathrm{Cii}= & \text { Package Cohesion } \\
\mathrm{Cij}= & \text { Package Cohesion }+ \text { Package Coupling } \\
& \quad \text { if } \mathrm{i}=\mathrm{j} \text { is Package Cohesion, } \\
& \quad \text { if } \mathrm{i} \neq \mathrm{j} \text { is Package Coupling) } \\
\mathrm{d}= & \text { number of package }
\end{aligned}
$$

\section{Formulation of Modularity Index}

Finally, the formulation of Modularity Index is the product of SA and the sum of all package quality in the software system as stated in the following formula:

$$
M_{I}=S_{A} \times \sum_{i=1}^{j} P_{Q i}
$$

Where:

$$
\begin{aligned}
& \mathrm{M}_{\mathrm{I}}=\text { Modularity Index } \\
& \mathrm{S}_{\mathrm{A}}=\text { Software Architecture Value } \\
& \mathrm{P}_{\mathrm{Qi}}=\text { Package Quality of Package i }
\end{aligned}
$$

The proposed modularity index is a quality metrics will have the following properties:

- It has no upper bound: the value of modularity index increases as the number of module / package increases.

- The value of modularity index, especially the value of $\mathrm{S}_{\mathrm{A}}$ depends on how the packages are coupled to each other. The limitation of connection of packages to only itself (package cohesion) or to only some dedicated packaged (e.g APIs, proxy, etc.) will improve the value of $S_{A}$.

\section{Case Study: JFreeCharT}

JFreeChart is a free $100 \%$ Java chart library that makes it easy for developers to display professional quality charts in their applications (http://www.jfree.org/jfreechart) . This projects is one of the 59 OSS Projects used for modularity index formulation. For this case study, this project is chosen because:

- $\quad$ High $\mathrm{S}_{\mathrm{A}}$ value (more than 0.7 since version 0.9.21)

- Relatively large number of packages (more than 30)

There are 52 versions available from the project's site, but only 33 are able to be analyzed using SONAR tool and being measured. The results are show in the following Fig.3.

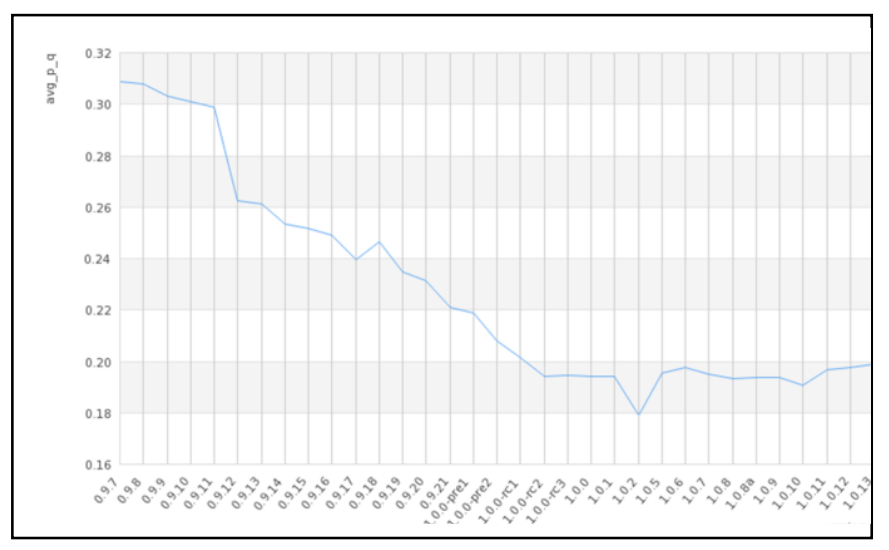

Figure 3. Average $\mathrm{P}_{\mathrm{Q}}$ in 33 versions of JFreeChart

Fig. 3 above shows that the average package quality of the JFreeChart over 33 versions are decreasing consistently. This indicates the problem in the quality of each classes in each packages, such as:

- increasing size of NCLOC in each class.

- increasing number of functions in class.

- decreasing number of LCOM4 (Cohesion Metrics) in class.

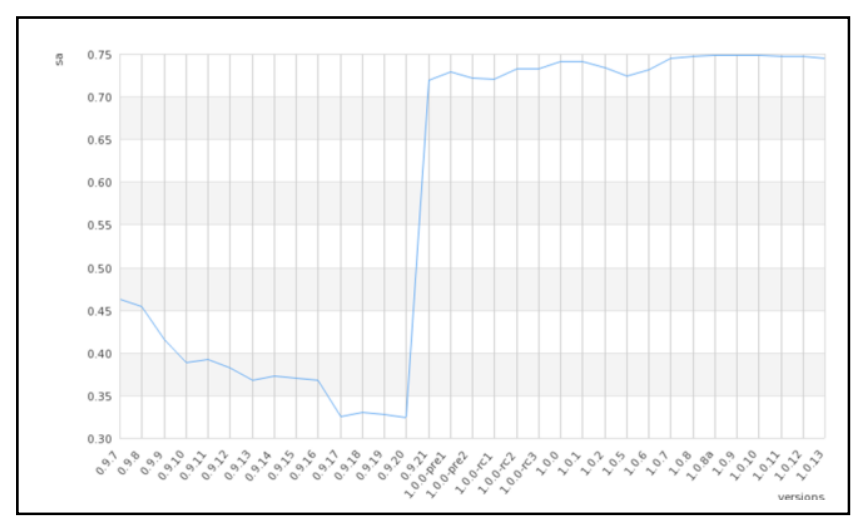

Figure 4. SA value in 33 versions of JFreeChart 
Fig. 4 above shows that the structure of software architecture is improving. After consistent decrease in SA value in early versions of the system, there seems to be significant effort conducted before the release of version 1.0.0 started from version 0.9.21. The system from version 0.9.21 onward showing high number of SA.

The modularity index itself is shown in Fig. 5. The figure is showing improvement by the factor of two from early versions (until version 0.9.20) and late versions (version 1.0.5 onwards). There are significant jump in the value of modularity index from version 0.9.21 until version 1.0.2 indicating the period of major restructuring of the system before the release of milestone version 1.0.0.

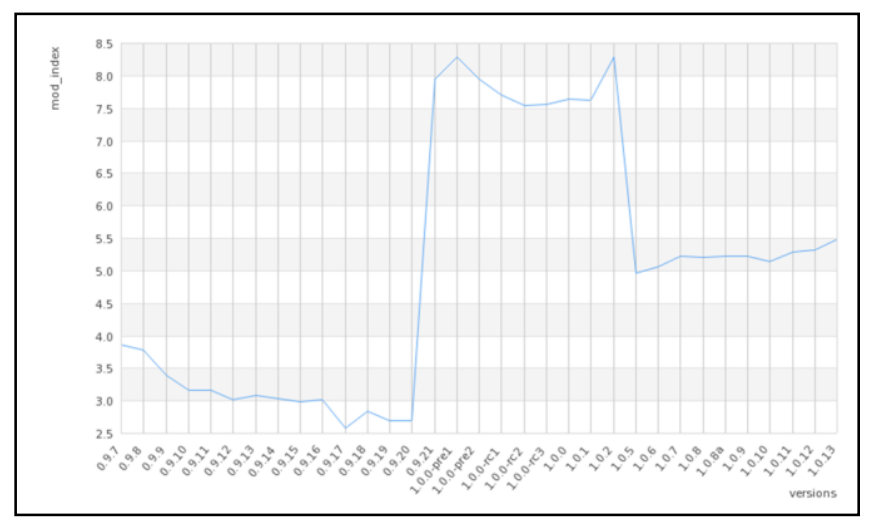

Figure 5. Modularity Index in 33 versions of JFreeChart

It can be seen from above case study that Modularity Index and its components $\left(\mathrm{P}_{\mathrm{Q}}\right.$ and $\left.\mathrm{S}_{\mathrm{A}}\right)$ are able to point the strength and potential problems in the development of JFreeChart OSS Projects. This information may give a valuable insight to the initiator and developers of the project in improving their project.

\section{CONCLUSION}

Open Source Software (OSS) Projects are now gaining popularity and becoming one alternatives in developing software. Despite the the many success story of OSS Projects such as Apache, Mozilla, etc., the fact the many more of these projects that are failed needs are alarming. Some studies have identified that modularity is one of the key success factors of OSS Projects and authors believe that implementing modularity approach since early start of the project will increase the success of the project. This paper presents the first quantitative measure of modularity for Java-based OSS Projects called modularity index.

The formulation of modularity index are performed by analyzing the software metrics attributing to modularity of 59 Java-based OSS Projects from sourceforge.net which have been downloaded more than $100 \mathrm{~K}$ times. By analyzing the similarity of these projects from class level, package level, and system level, the modularity index are formulated. As the validation of the software metrics, 33 out of 52 versions of JFreeChart OSS projects are analyzed using this metrics and the metrics are able to identify the strength and potential problems of the project.
Future study relating to this metrics involve further validation and integration into a framework called modularity framework in which the measurement of Modularity Frameworks will generate recommendations for improvement during OSS project's development. The integration of the software metrics into a web-based IDE will provide useful tool for project initiators and developers in improving their OSS Projects.

\section{ACKNOWLEDGMENT}

The authors would like to thank Maranatha Christian University (http://www.maranatha.edu) that provides funding for the research, and the Department of Computer Science and Electronics, Universitas Gadjah Mada (http://mkom.ugm.ac.id) that provides technical support for the research.

\section{REFERENCES}

[1] Ammar H., Shereshevsky M., Mili A., Rabie W., Radetsky N. (2008), "Software architecture metrics", Seminar Presentation, Faculty of Information Science \& Engineering, Management \& Science University, Shah Alam, Malaysia, May 12, 2008. Available: http://www.docstoc.com/docs/6802629/Software-Architecture-Metrics

[2] Aruna M., M.P. Suguna Devi M.P, Deepa M. (2008), "Measuring the quality of software modularization using coupling-based structural metrics for an OSS system", Proceeding of the First International Conference on Emerging Trends in Engineering and Technology 2008

[3] Asundi J. (2007), "The need for effort estimation model for open source software projects", Proceeding of the Open Source Application Workspace: Fifth Workshop on Open Source Sofware Engineering 2007

[4] Bouktif S., Antoniol G., Merlo E. (2006), "A feedback based quality assessment to support open source software evolution: the GRASS case study", 22nd IEEE International Conference on Software Maintenance 2006, pp 155 - 165

[5] Cai Y., Huynh S. (2007), "An evolution model for software modularity assessment", Proceeding of the Fifth International Workshop on Software Qualty 2007 (WoSQ'07).

[6] Capiluppi A., Ramil J.F. (2004), "Studying the evolution of open source systems at different levels of granularity: two case studies", Proceeding on the $7^{\text {th }}$ IEEE International Workshop of Principles of Software Evolution, 2004, pp 113 - 118.

[7] Capra E., Francalanci C., Merlo F. (2008), "An empirical study on the relationship among software design quality, development effort, and governance in open source projects", IEEE Transactions on Software Engineering Vol. 34, No. 6, Nov/Dec 2008, pp 765 - 782.

[8] Chidamber S.R., Kemerer C.F. (1994), "Metrics suite for object oriented design", IEEE Transaction on Software Engineering, Vol. 20 No. 6 June 1994, pp 476 - 493.

[9] Christley S., Madey G. (2007), "Analysis of activity in the open source software development community", Proceeding of the 40th IEEE Annual Hawaii International Conference on System Sciences, 2007, pp $166 b$.

[10] Crowston K., Wei K., Li Q., Howison J. (2006), "Core and periphery in free / libre and open source software team communications", Proceeding of the 39th IEEE Hawaii International Conference on System Sciences 2006

[11] DeKoenigsberg G. (2008), "How successful open source projects work, and how and why to introduce students to the open source world", 21 st IEEE Conference on Software Engineering Education and Training, 2008, pp $274-276$.

[12] Dinh-Trong T., Bieman J.M. (2004), "Open source software development: a case study of FreeBSD", Proceedings of the 10th IEEE International Symposium on Software Metrics, 2004, pp 96 - 105.

[13] Ellis H.J.C., Morelli R.A. , Lanerolle T.R., Damon J., Raye J. (2007), "Can humanitarian open-source software development draw new sudents to CS?", Proceeding of the 38th SIGCSE Technical Symposium on Computer Science Education 2007, pp 551 - 555. 
[14] Emanuel A.W.R, Wardoyo R., Istiyanto J.E., Mustofa K. (2011), "Statistical analysis on software metrics affecting modularity in open source software", International Journal of Computer Science and Information Technology (IJCSIT), Vol. 3, No. 3, June 2011, pp 105 118

[15] Emanuel A.W.R, Wardoyo R., Istiyanto J.E., Mustofa K. (2010), "Success rules of OSS projects using datamining 3-itemset association rule", International Journal of Computer Science Issue (IJCSI), Vol. 7 Issue 6 Nov. 2010, pp $71-80$.

[16] Emanuel A.W.R, Wardoyo R., Istiyanto J.E., Mustofa K. (2010), "Success factors of OSS projects from sourceforge using datamining association rule", Proceeding of 2010 International Conference on Distributed Frameworks for Multimedia Applications (DFmA) 2010, pp $141-148$

[17] Gurbani V.K., Garvert A., Herbsleb J.D. (2005), “A case study of open source tools and practices in commercial setting", Proceeding of the fifth Workshop on Open Source Software Engineering 2005, pp 1 - 6.

[18] Hahsler M. (2005), "A quantitative study of the adoption of design patterns by open source software developers", Chapter V of Free / Open Source Software Development by Stefan Koch, Idea Group Publishing, ISBN 1-59140-371-5, 2005, pp $103-123$.

[19] Hitz M., Montazeri B. (1995), "Measuring coupling and cohesion in object-oriented systems", Proceeding International Symposium on Applied Corporate Computing, Oct. 25-27 1995, Monterrey, Mexico, 75-76, 197, 78-84

[20] Lawrie T., Gacek C. (2002), "Issues of dependability in open source software development", Software Engineering Notes vol 27 no 3 of ACM Sigsoft. May 2002. Pp 34 -37

[21] Lee Y., Yang Y., Chang K.H. (2007), "Metrics \& evolution in open source software", Proceeding on Seventh International Conference on Quality Software - 2007

[22] Li P.L., Herbsleb J., Shaw M. (2005), “ Finding predictors of field defects for open source software systems in commonly available data sources: a case study of OpenBSD", Proceeding of 11th IEEE International Software Metrics Symposium, 2005, 32.

[23] McCabe T. (1976), "A complexity measure", IEEE Transactions On Software Engineering, Vol. Se-2, No. 4, December 1976, pp. 308-320.

[24] Melton H., Tempero E. (2007), "Toward assessing modularity", Proceeding of the First International Workshop on Assessment of Contemporary Modularization Techniques 2007 (ACoM'07)

[25] Meyer B., Oriol M., \& Schoeller B. (2009), "Software engineering: lecture 17-18: estimation techniques and software metrics", Chair of Software Engineering Website, available: http://se.inf.ethz.ch/teaching/2008-S/se-0204/slides/15-Estimation-andmetrics-1-6x.pdf , accessed: 18 January 2009

[26] Mockus A., Fielding R.T., Herbsleb J.(2002), "Two case studies of open source software development: apache and mozilla", ACM Transaction on Software Engineering and Methodology Vol. II No. 3, Juli 2002, 309 $-346$

[27] Mockus A, Fielding R.T, Herbsleb J.(2000), "A case study of open source software development: the apache server", ACM ICSE, 2000, $263-272$

[28] Munelly J., Fritsch S., Clarke S. (2007). “An aspect-oriented approach to the modularisation of context". Proceedings of the Fifth Annual IEEE International Conference on Pervasive Computing and Communication (PerCom'07)

[29] Nakagawa E.Y, de Sousa E.P.M., de Britto Murata K. (2008), "Software architecture relevance in open source software evolution: a case study", Annual IEEE International Computer Software and Application Conference, 2008, pp $1234-1239$.
[30] Paech B, Reuschenbach B (2006), “Open source requirements engineering", Proceeding of 14th IEEE International Requirement Engineering Conference: 257 - 262

[31] Raymond E.S. (2000), "The cathedral and the bazaar", version 3, Thyrsus Enterprises (http://www.tuxedo.org/ esr/), 2000.

[32] Spaeth S., Stuermer M. (2007), "Sampling in open source development: the case for using the debian GNU/linux dstribution", Proceedings of the 40th IEEE Hawaii International Conference on System Sciences, 2007, pp 166a.

[33] Stallman R. (1992), "Why software should be free", GNU Websites, 24 April 1992, Available: http://www.gnu.org/philosophy/shouldbefree.html

[34] Stewart K. J., Darcy D.P., Daniel S.L. (2005), "Observations on patterns of development in open source software projects", Proceeding on the fifth Workshop on Open Source Software Engineering 2005, pp 1 - 5.

[35] von Krogh G., Spaeth S., Haefliger S. (2005), "Knowledge reuse in open source software: an exploratory study of 15 open source projects", Proceeding of 38th Hawaii International Conference on System Sciences, 2005, pp. 198b

[36] Wang Y., Shao J. (2003), "Measurement of the cognitive functional complexity of software", Proceedings of the Second IEEE International Conference on Cognitive Informatics 2003 (ICCI'03).

[37] Zhou F., Davis J. (2008), "A model of bug dynamics for open source software", The Second IEEE International Conference on Secure System Integration and Reliability Improvement 2008, pp 185 - 186.

\section{AUTHORS PROFILE}

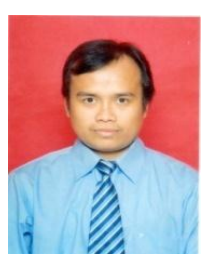

Andi Wahju Rahardjo Emanuel is a Full Time Lecturer at the Bachelor Informatics Program, Faculty of Information Technology, Maranatha Christian University in Bandung, Indonesia. He is graduated as BSEE in Purdue University, Indiana, USA in 1996 and MSSE in The University of Melbourne in 2001. He is currently taking his Doctoral Program at the Department of Computer Science and Electronics, Gadjah Mada University in Yogyakarta, Indonesia

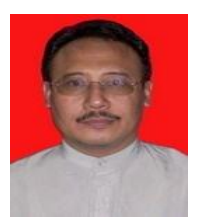

Retantyo Wardoyo is an Associate Professor at the Department of Computer Science and Electronics, Universitas Gadjah Mada in Yogyakarta, Indonesia. He is graduated as Bachelor of Mathematics in Gadjah Mada University, Indonesia . He received his M.Sc in Computer Science in University of Manchester, UK and received his $\mathrm{PhD}$ in Computation in University of Manchester Institute of Science and Technology, UK.

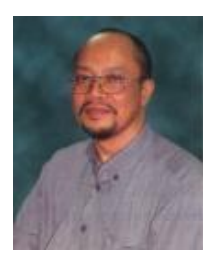

Jazi Eko Istiyanto is a Professor and Head of the Department of Computer Science and Electronics, Universitas Gadjah Mada in Yogyakarta, Indonesia. $\mathrm{He}$ is graduated as Bachelor of Physics in Gadjah Mada University, Indonesia. He gets his Postgraduate Diploma (Computer Programming and Microprocessor), M.Sc (Computer Science) and PhD (Electronic System Engineering) at University of Essex, UK.

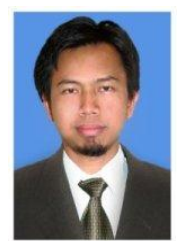

Khabib Mustofa is an Assistant Professor at the Department of Computer Science and Electronics, Universitas Gadjah Mada in Yogyakarta, Indonesia. $\mathrm{He}$ is graduated as Bachelor of Computer and Master of Computer at Gadjah Mada University, Indonesia. $\mathrm{He}$ receives his Dr. Tech in Computer Science at The Vienna University of Technology, Austria. 